East African Medical Journal Vol. 86 No. 7 July 2009

ADHERENCE TO FEEDING GUIDELINES AMONG HIV-INFECTED AND HIV UNINFECTED MOTHERS IN A RURAL DISTRICT IN UGANDA

J. N. Babirye, MBChB, MPH, Assistant Lecturer, F. Nuwaha, MD, PhD, Associate Professor, Department of Disease Control and Environmental Health Sciences, Makerere University School of Public Health, P.O. Box 7072, Kampala, Uganda and A. E. Grulich, MBBS, PhD, Professor and Head, HIV Epidemiology and Prevention Programme, National Centre in HIV Epidemiology and Clinical Research, Level 2, 376 Victoria Street, Darlinghurst, NSW 2010

Request for reprints to: Dr. J. N. Babirye, Department of Disease Control and Environmental Health Sciences, Makerere University School of Public Health, P. O. Box 7072, Kampala, Uganda

\title{
ADHERENCE TO FEEDING GUIDELINES AMONG HIV-INFECTED AND HIV UNINFECTED MOTHERS IN A RURAL DISTRICT IN UGANDA
}

\author{
J. N. BABIRYE, F. NUWAHA and A. E. GRULICH
}

\begin{abstract}
Objective: To describe the infant feeding behaviour of HIV-infected and HIV-uninfected mothers, and identify factors influencing adherence to infant feeding guidelines.

Design: Analytical cross-sectional study.

Setting: Bushenyi, rural district in South-western Uganda

Participants: One hundred and ninety four mothers who had a child less than 12 months of age. About half, $94(48.5 \%)$, of these were HIV -infected.

Main outcome measures: Proportion of mothers who exclusively breastfed, complementary fed, replacement fed, and adhered to feeding guidelines.

Results: Most $(84.5 \%, 164 / 194)$ of the mothers had ever breastfed their infants, the rest had exclusively replacement fed since birth. Among children less than six months who were breastfeeding, $31.5 \%$ (34/108) were exclusively breastfeeding and the rest were mixed feeding. HIV-infected mothers were more likely than HIV-uninfected mothers to exclusively breastfeed (Crude Odds Ratio [COR], 3.61, 95\% Confidence Interval [CI] 1.42-9.21). For infants older than six months, complementary feeding was more common among HIV-uninfected $(100 \%)$ than HIV-infected mothers $(41.7 \%$; $<<0.001)$. Among infants of all ages, none of the HIV-uninfected and $45 \%$ of HIV-infected mothers were replacement feeding $(\mathrm{p}<0.001)$. More than a half $(59.8 \%)$ of the mothers adhered to infant feeding guidelines. The only independent predictor of adherence after multivariate analysis was mother ever attending infant feeding counselling (AOR 9.03; 95\% CI 4.03-20.25). Only $35 \%$ of mothers reported ever attending infant feeding counselling. Conclusions: Infant feeding counselling was associated with improved adherence to feeding guidelines. Since infant feeding counselling is low in this population there is need for scale-up of this essential service.
\end{abstract}

\section{INTRODUCTION}

Breastfeeding is one of the low cost technologies promoting health and survival of children especially in low income countries (1). The HIV pandemic has however made recommendations aboutbreastfeeding problematic especially in high HIV-prevalent countries of sub-Saharan Africa. HIV transmission may occur through breastfeeding from mother-tochild (MTCT) in HIV-infected mothers and the risk of transmission decreases if exclusive breastfeeding (EBF) or replacement feeding is practiced. On the other hand replacement feeding increases childhood mortality and morbidity (1-3). Thus infant feeding guidelines recommend that HIV infected women should practice replacement feeding from birth if this is affordable, feasible and safe; otherwise EBF is recommended for the first six months and replacement feeding thereafter. However current guidelines for HIV-infected mothers are pragmatic and recognise that EBF followed by replacement feeding may not be feasible and therefore state that complementary feeding may be considered where replacement feeding is impracticable $(2,3)$. For HIV-uninfected mothers and mothers of unknown HIV status, the guidelines state that breastfeeding should continue for 24 months with additional (complementary) feeds being introduced at six months. 
Available evidence suggests that without intervention adherence to these infant feeding guidelines is low (4-6). The proportion of infants being EBF by six months ranges between $6-26 \%$ (47). Besides there have been concerns that as access to programmes aimed at preventing mother-to-child transmission of HIV (PMTCT) increaseinsub-Saharan Africa there could be confusion regarding information meant for HIV infected mothers and HIV uninfected mothers with antecedent uninfected influence on adherence to guidelines.

In this study, we describe the infant feeding behaviour of HIV-infected and HIV-uninfected mothers, and identify factors influencing adherence to infant feeding guidelines in a rural district of Uganda where a PMTCT programme has been running for five years.

Methods: The study was conducted in Bushenyi in South-western Uganda. Bushenyi is a rural district with a land area of about 3949 Square Kilometres and a total population of 739,000 people (projected from 2002 census). The people are predominantly peasant farmers whose mainoccupation is subsistence agriculture. Three quarters ( $77 \%$ ) of the population is within 5 kilometres of a health facility. Health services in the district are offered by 98 health facilities and 215 immunisation posts.

Ten sites for prevention of mother to child transmission of HIV (PMTCT) were started in January 2003. Between March and December 2003,9406 women (constituting about $30 \%$ of all antenatal attendees) received pre- test HIV counselling, 2728 $(29 \%)$ were tested and $235(8.6 \%)$ of them were HIVinfected.

All mothers who attend antenatal care (irrespective of their HIV status) in the district are supposed to receive infant feeding counselling. The messages regarding infant feeding are supposed to be reinforced during attendance at immunisation but this is rarely done. A possible reason for this is that the counsellors at these sites conduct the pre- and post-test HIV counselling and offer infant feeding counselling, in addition to the immunisation, antenatal care and midwifery duties at the health care facilities. Moreover, all the sites have one or two counsellors.

Sampling and eligibility criteria: From January to July 2004 consecutive eligible mothers at ten static immunisation posts located at health centres with PMTCT services in Bushenyi district were approached for study inclusion. Mothers were eligible for study inclusionif they were aged $18-49$ years, had previously undertaken and received an HIV-test result during the most recent pregnancy, had infants younger than 12 months, and gave written consent to participate. The study aimed at recruiting about 172 mothers with about $50 \%$ of them being HIV -infected. This sample size was enough to compare adherence to infant feeding guidelines among HIV-infected and HIV-uninfected mothers with $80 \%$ power, 95\% confidence interval at an odds ratio of 3 if the prevalence of adherence among HIV-uninfected mothers is about $20 \%$ (statcal, sample size calculator for comparative cross-sectional studies, EPIinfo, CDC Atlanta, 2003). We were able to obtain equal numbers in both categories of mothers even though the HIV prevalence and HIV-test uptake was relatively low in this community during the study period. HIV infected mothers tended to be more frequent than usual at the health units because at the time of the study, a local NGO; "Integrated community based initiative" (ICOBI) was supporting the follow up of HIV -infected mothers after delivery. The visits were scheduled to match the usual immunisation programme to avoid any possible identification of the HIV status of the mother.

Data collection: A semi-structured pretested interview schedule was utilised to collect data on feeding behaviour. More data was collected on sociodemographic features such as maternal age, parity, occupation, formal education, religion, residential address, marital status; partner support, HIV status, infant age, sex, and position of the infant, knowledge of MTCT, knowledge of PMTCT, having received infant feeding counselling at least once. The WHO assessment tool for research on breastfeeding and replacement feeding was adopted and included in the interview schedule to collect data on current infant feeding behaviour (8).

The interview schedule was translated into the local language (Runyankore) and back translated into English for clarity in meaning. The schedules were reviewed daily for completeness and corrections made where necessary. As a quality control measure $5 \%$ of all interviews were repeated on the same day as the original interview. Any discrepancies in the responses recorded were then reconciled and this sometimes involved a visit to the home of the interviewee.

Study definitions: Current infant feeding practice was based on a detailed account of what the mother had been feeding the infant during the 24 hours (24-hour recall) prior to the interview and in the previous week (seven day recall). Exclusive breastfeeding was defined as breastfeeding and giving the infant no other food or drink (including water). Drops or syrups consisting of vitamins, mineral supplements or prescribed medicines were allowed (8).

Complementary feeding was defined as feeding that involved giving any food to infants who were six or more months old, whether manufactured or locally prepared in addition to breastfeeding. Replacement feeding included those mothers feeding a child with 
another dietbut not receiving any breast milk regardless of the age of the infant. Predominant breastfeeding and / or mixed feeding denoted breastfeeding a baby, but also giving water or water-based drinks, such as tea, even in small amounts and this occurs in infants aged less than six months (8).

Data analysis: All interview schedules were double entered and analysed using SPSS version 12.0.1 (SPSS Inc. Chicago, Illinois). Statistically significant response patterns were considered if a two sided p-value was $<0.05$. To compare feeding behaviour among HIV -infected and HIV uninfected mothers, Chi-square tests $\left(\mathrm{X}^{2}\right)$ or Crude odds ratios (COR) were used to compare categorical data whereas F-ratios were used to compare continuous data.

Mothers who adhered to infant feeding guidelines were compared to those who did not adhere to the guidelines using logistic regression at univariate and multivariate levels. All variables that influenced adherence to guidelines were considered in multivariate analysis using the forward stepwise approach after considering multi-collinearity of the variables. Adjusted odds ratios (AOR) and their 95\% Confidence intervals were used toidentify predictors of adherence to infant feeding guidelines.

Ethics: This study was approved by the Human Research Ethics Committee of the University of New South Wales, Sydney. Independent ethics approval was also obtained from the Higher Degrees Research and Ethics Committee of the Makerere University School of Public Health, and from the Uganda National Council for Science and Technology.

Results: One hundred and ninety four respondents comprising $100 \mathrm{HIV}$-uninfected and $94 \mathrm{HIV}$-infected mothers participated in the study. Overall, the HIV-infected and HIV-uninfected mothers in this study were comparable in a range of demographic characteristics except that HIV-infected mothers were older (Table 1).

Table 1

Socio-demographic characteristics of the HIV-infected and HIV-uninfected mothers

\begin{tabular}{|c|c|c|c|c|c|}
\hline \multirow{3}{*}{ Characteristic } & \multicolumn{4}{|c|}{ HIV status } & \multirow[b]{3}{*}{ F-ratio(df) } \\
\hline & \multirow{2}{*}{\multicolumn{2}{|c|}{$\begin{array}{l}\text { Infected } \\
\text { Mean (SD) }\end{array}$}} & \multirow{2}{*}{\multicolumn{2}{|c|}{$\begin{array}{l}\text { Uninfected } \\
\text { Mean (SD) }\end{array}$}} & \\
\hline & & & & & \\
\hline Age $(n=194)$ & \multicolumn{2}{|c|}{$27.9(4.93)$} & \multicolumn{2}{|c|}{$25.1(5.08)$} & $1.14(192)$ \\
\hline Parity $(\mathrm{n}=176)$ & \multicolumn{2}{|c|}{$3.0(1.66)$} & \multicolumn{2}{|c|}{$2.9(1.87)$} & $1.83(174)$ \\
\hline Age of infant in months $(n=194)$ & \multicolumn{2}{|c|}{$3.8(3.25)$} & \multicolumn{2}{|c|}{$3.6(3.00)$} & $0.77(192)$ \\
\hline Age at first marriage in years $(n=171)$ & \multicolumn{2}{|c|}{$19.7(3.27)$} & \multicolumn{2}{|c|}{$18.8(3.00)$} & $0.27(169)$ \\
\hline \multirow[t]{2}{*}{ Length lived at residence in years $(n=194)$} & \multicolumn{2}{|c|}{$8.4(7.37)$} & \multicolumn{2}{|c|}{$7.6(8.74)$} & $2.75(192)$ \\
\hline & No. & $(\%)$ & No. & $(\%)$ & $X^{2} \quad(d f)$ \\
\hline \multicolumn{6}{|l|}{ Maternal education $(\mathrm{n}=194)$} \\
\hline None/primary & 69 & 73.4 & 80 & 80.0 & \\
\hline Secondary/tertiary & 25 & 26.6 & 20 & 20.0 & $0.85 \quad(1)$ \\
\hline \multicolumn{6}{|l|}{ Maternal religion $(\mathrm{n}=193)^{2}$} \\
\hline Muslim & 10 & 10.8 & 8 & 8.0 & \\
\hline Catholic & 35 & 37.6 & 40 & 40.0 & \\
\hline Protestant & 48 & 51.6 & 52 & 52.0 & $0.46 \quad(2)$ \\
\hline \multicolumn{6}{|l|}{ Maternal occupation $(n=191)$} \\
\hline Work away from home & 34 & 37.0 & 25 & 25.3 & \\
\hline Home based & 58 & 63.0 & 74 & 74.7 & $2.54 \quad(1)$ \\
\hline \multicolumn{6}{|l|}{ Mother has partner $(\mathrm{n}=194)$} \\
\hline Yes & 66 & 70.2 & 80 & 80.0 & \\
\hline No & 28 & 29.8 & 20 & 20.0 & $1.99 \quad(1)$ \\
\hline \multicolumn{6}{|l|}{ Position of youngest child $(\mathrm{n}=176)^{3}$} \\
\hline First born & 18 & 20.2 & 29 & 33.3 & \\
\hline Others & 71 & 79.8 & 58 & 66.7 & $3.22 \quad(1)$ \\
\hline
\end{tabular}




\begin{tabular}{|c|c|c|c|c|c|}
\hline \multicolumn{6}{|c|}{ Infant sex $(\mathrm{n}=187)^{4}$} \\
\hline Female & 44 & 50.0 & 45 & 46.4 & \\
\hline Male & 44 & 50.0 & 52 & 53.6 & $0.12(1)$ \\
\hline \multicolumn{6}{|c|}{ Infant age $(\mathrm{n}=194)$} \\
\hline$<6$ months & 71 & 75.5 & 79 & 79.0 & \\
\hline$\geq 6$ months & 23 & 24.5 & 21 & 21.0 & $0.16(1)$ \\
\hline
\end{tabular}

${ }^{1} \mathrm{p}$-value defines the difference between HIV-infected and HIV-uninfected respondents.

${ }^{2}$ Data on religion was missing in one HIV -infected participant.

${ }^{3}$ Data on position of the youngest child was missing for $18(9.3 \%)$ participants (13 were among HIV-uninfected respondents).

${ }^{4}$ Two of the HIV-uninfected mothers had twins of different sexes and data on the sex of the youngest infant was missing for seven (3.6\%) respondents.

df denotes degree of freedom.

Only 35.4\% (69/194) of all mothers reported ever attending infant feeding counselling and HIVinfected $(70 \%)$ mothers were more likely than HIV-uninfected women (3\%) to receive counselling (Crude odds ratio (COR), 76.2, 95\% confidence interval (CI) 21.7-395.6). Most (84.5\%, 164/194) of the mothers had ever breastfed their infants, the rest had exclusively replacement fed since birth. Most $(78.4 \%, 152 / 194)$ of the mothers were currently breastfeeding, the rest were replacement feeding. All the HIV-uninfected mothers compared to about half $(55.3 \% ; 52 / 94)$ of the HIV-infected women were breastfeeding $(\mathrm{p}<0.001)$.
Three quarters $(77.3 \%, 150 / 194)$ of the mothers in this study had infants younger than six months and among those breastfeeding in this category $31.5 \%$ (34/1 08) were exclusively breastfeeding whereas $68.5 \%(74 / 108)$ were mixed feeding. HIV-infected mothers $(48.8 \%)$ were almost four times more likely than HIV-uninfected mothers (20.9\%) to exclusively breastfeed their infants (COR 3.61,95\% CI 1.429.21). About half $(51.2 \%)$ of breast feeding children younger than six months and born to HIV -infected women compared to $79.1 \%$ of similar infants among HIV-uninfected mothers were mixed breastfeeding $(\mathrm{p}<0.004)$ (Table 2).

Table 2

Infant feeding behaviour among HIV-infected and HIV-uninfected mothers

\begin{tabular}{|c|c|c|c|c|c|c|}
\hline \multirow[t]{3}{*}{ Characteristic infected } & \multicolumn{4}{|c|}{ HIV status } & \multirow[t]{3}{*}{$X^{2} \quad(\mathrm{df})$} & \multirow[t]{3}{*}{ P-value ${ }^{1}$} \\
\hline & \multicolumn{2}{|c|}{ Infected } & \multicolumn{2}{|c|}{ Uninfected } & & \\
\hline & No. & $(\%)$ & No. & $(\%)$ & & \\
\hline \multicolumn{7}{|c|}{ Mother has ever breastfed the infant $(n=194)$} \\
\hline Yes & 64 & 68.1 & 100 & 100 & $35.35(1)$ & $<0.001$ \\
\hline No & 30 & 31.9 & 0 & 0.0 & & \\
\hline \multicolumn{7}{|c|}{ Current infant feeding practice $(\mathrm{n}=194)$} \\
\hline Breastfeeding & 52 & 55.3 & 100 & 100 & $54.42(1)$ & $<0.001$ \\
\hline Replacement feeding & 42 & 44.7 & 0 & 0.0 & & \\
\hline \multicolumn{7}{|c|}{ Breastfeeding among infants $<6$ months $(\mathrm{n}=108)^{2}$} \\
\hline Exclusive breastfeeding & 20 & 48.8 & 14 & 20.9 & $7.92(1)$ & 0.004 \\
\hline Mixed breastfeeding & 21 & 51.2 & 53 & 79.1 & & \\
\hline \multicolumn{7}{|c|}{ Current infant feeding among infants $\geq 6$ months $(n=48)$} \\
\hline Replacement feeding & 14 & 58.3 & 0 & 0.0 & $17.04(1)$ & $<0.001$ \\
\hline Complementary feeding & 10 & 41.7 & 24 & 100 & & \\
\hline \multicolumn{7}{|c|}{${ }^{1}$ P-value defines the difference between HIV-infected and HIV-uninfected respondents } \\
\hline \multicolumn{7}{|c|}{$\begin{array}{l}2 \text { Due to missing data } 10(5.2 \%) \text { breastfeeding respondents could not be classified into any of the } \\
\text { above categories of infant feeding (nine were HIV-uninfected and one was HIV-infected). Therefore } \\
\text { these mothers were excluded from any subsequent analysis. }\end{array}$} \\
\hline
\end{tabular}


Among infants older than six months 70.8\% (34/48) were complementary feeding and the rest were replacement feeding (Table 2). HIV-uninfected mothers $(100 \%)$ were more likely than HIV-infected mothers $(41.7 \%)$ to complementary feed $(p<0.001)$. Eighty one percent of the HIV infected mothers who were replacement feeding reported that they had attended infant feeding counselling and two thirds $(71 \%)$ of these reported choosing this mode during counselling. However, only $7 \%$ of the mothers had ever seen a practical demonstration on mixing the infant feeds.

Adherence to Ugandanfeeding guidelines: Mothers were categorised into two categories based on whether they were feeding according to Ugandan guidelines (adherent) or not (non-adherent). Adherent HIVinfected mothers were those whose children were below six months and were exclusively breastfeeding $(n=20)$, had children older than six months and were complementary feeding $(n=10)$ or those whopracticed exclusive replacement feeding regardless of the age of the infant $(n=42)$. And an HIV -infected mother was classified as non-adherent if she was, mixed feeding $(n=21)$ at the time of interview.

An HIV-uninfected mother was defined as adherent if she exclusively breastfed during the first six months $(n=14)$ or complementary fed at and after six months $(n=24)$. The non-adherent HIV-uninfected mothers were those who mixed fed $(n=53)$ or exclusively replacement fed $(n=0)$. Thus $110 / 184(59.8 \%)$ of mothers were adherent to Ugandan feeding guidelines. Statistically significant variables associated with adherence at univariate analysis are shown in Table 3.

Table 3

Univariate analysis of factors associated with adherence to Ugandan feeding guidelines

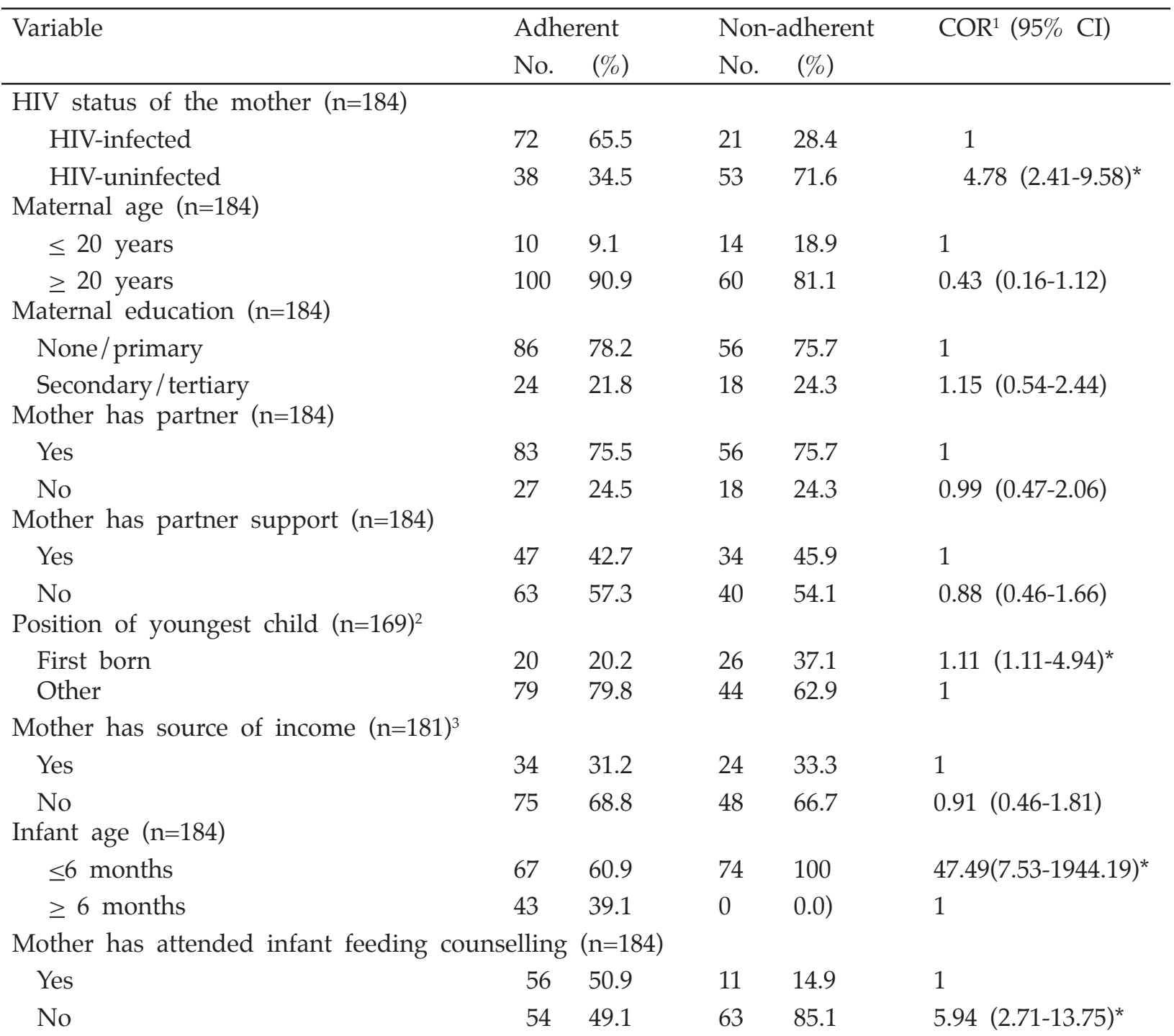




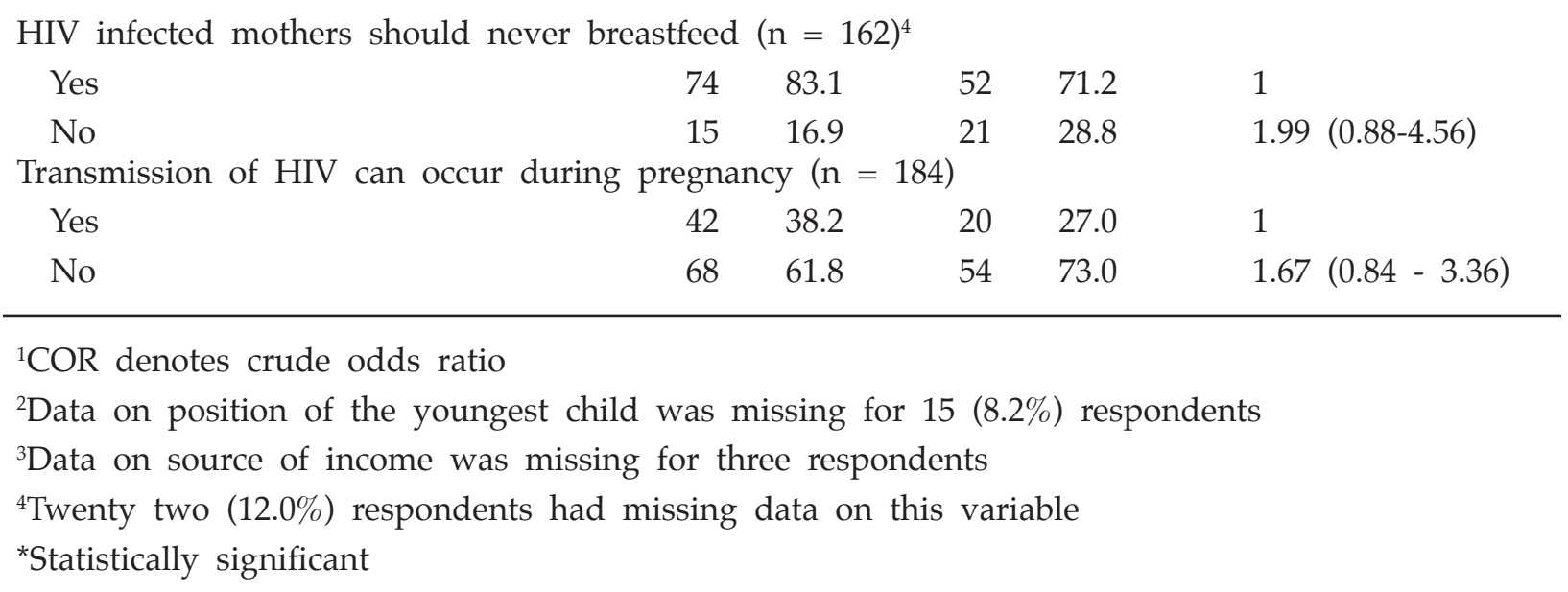

At univariate analysis adherence to infant feeding guidelines was increased by mother being HIVinfected, having had a child before, infant being older than six months, and ever attending infant feeding counselling. The socio- demographic features of the mother such as age, occupation, address, length lived at address, formal education, religion, marital status, age at first marriage, support of the partner, sex of the youngest infant, maternal attitude towards breastfeeding among HIV -infected women did not influence adherence to infant feeding guidelines. In addition, knowledge on timing, route of MTCT, and methods for PMTCT and its timing were not associated with adherence to feeding guidelines. At multivariate analysis, the only independent predictor of adherence was mother ever attending infant feeding counselling (AOR 9.03; 95\% CI 4.0320.25). This variable could explain about $50.8 \%$ of the variance regarding adherence to infant feeding guidelines. HIV status of mother that was significant at univariate analysis became insignificant after multivariate analysis.

\section{DISCUSSION}

The first major finding of our study was that about half $(59.8 \%)$ of mothers were adherent to Ugandan infant feeding guidelines with most of the non-adherence to infant feeding guidelines being contributed to by the very high proportion of mothers who practice mixed feeding in the first six months of life. These results tend to agree with the reported low adherence to infant guidelines in much of sub-Saharan Africa whether under optimal research conditions $(9,10)$ or under routine field settings (11-14). The reasons proposed for the widespread mixed breastfeeding practices among African mothers include traditional beliefs that reject the suitability of colostrum as an adequate complete early feed (15), mothers' perceptions of 'insufficient milk' and an unsatisfied baby (12), as well as mothers working away from home $(12,15)$.
In our study, adherence to infant feeding guidelines was mainly influenced by attendance at infant feeding counselling. Although only 35\% of the mothers had ever received infant feeding counselling. HIV infected mothers are more likely to adhere to infant feeding guidelines at univariate' analysis although this higher likelihood disappears at multivariate analysis when controlled for exposure to feeding counselling. A possible explanation for this observation is that HIV-infected mothers are more likely to be counselled compared to HIV -uninfected mothers or mothers of unknown HIV status. Thus the effect of HIV status on adherence to infant feeding guidelines is more likely mediated through attendance at infant feeding counselling. The observation that infant feeding counselling greatly improves adherence to infant feeding guidelines has been reported elsewhere in intervention randomised studies in Bangladesh and Zimbabwe $(4,5)$.

In our study setting, infant feeding counselling was reported almost exclusively among HIV-infected women. This is probably due to the heavy work load among the counsellors as mentioned earlier in this report. Consequently, the counselling tends to be insufficient in quality and quantity with almost no follow-up at immunisation centres or within communities and no demonstration of mixing of infant feeds. These observations have implications for policy, practice, and further research. The severe shortage of health workers is likely to continue in the foreseeable future in Uganda and other low income countries. Remedial action that has been suggested may be to consider non-medical personnel including experienced mothers to be recruited as infant feeding counsellors (5).

Furthermore, additional data is required on how the quality and quantity of infant feeding counselling (to all mothers regardless of their HIV status) could be improved and on how community programmes aimed at ongoing feeding counselling could be implemented. More intervention studies aimed at 
improving adherence to infant feeding guidelines are also needed.

In interpreting these results, some limitations of the study design should be considered. First, a differential error in measurement could have arisen if HIV-infected mothers were less likely to report the use of sub-optimal modes of feeding that lead to HIV transmission because of perceived' unacceptability of sub-optimal feeding behaviour after receiving infant feeding counselling. Second, we assessed feeding behaviour in the seven days prior to interview. This overlooks the varying nature of feeding behaviour as the infant grows. Although a seven day maternal recall has been used to accurately measure feeding practices since birth in areas of high HIV prevalence (16), current feeding practice does not necessarily predict previous or future feeding patterns.

\section{ACKNOWLEDGEMENTS}

To the research assistants Mrs. E. Talinyeeba and Mrs. J. Bithire who interviewed the mothers. Grateful to the medical providers at each PMTCT site that assisted us in referring eligible mothers and to the kind guidance of the PMTCT coordinator Dr. S. Asiimwe who gave insights into the Bushenyi PMTCT programme. This study was supported by a grant from the Australian government.

\section{REFERENCES}

1. WHO Collaborative Study Team on the Role of Breastfeeding on the Prevention of Infant Mortality. Effect of breastfeeding on infant and child mortality due to infectious diseases in less developed countries: a pooled analysis. Lancet. 2000; 355: 451-455.

2. Ugandan Ministry of Health. Policy guidelines on feeding of infants and young children in the context of HIV / AIDS. Kampala, 2001.

3. WHO. HIV and Infant Feeding Technical Consultation held on behalf of the interagency task team on prevention of HIV infections in pregnant women, mothers and their infants. Geneva: WHO; 2006. Available at:http: / / www.who.intireproductivehealthlst is/mtct/infantfeedingconsensusstatement.pdf. Accessed: April 10, 2008.

4. Piwoz, E.G., Iliff, P.J., Tavengwa, N. etal. Aneducation and counselling program for preventing breastfeeding-associated HIV transmission in Zimbabwe: design and impact on maternal knowledge and behaviour. J. Nutr. 2005; 135: 950-955.
5. Haider, R., Ashworth, A., Kabir, I., et al. Effect of community-based peer counsellors on exclusive breastfeeding practices in Dhaka, Bangladesh: a randomised controlled trial. Lancet. 2000; 356:16431647.

6. Bhandari, N., Bahl, R., Mazumdar, S. et al. Infant Feeding Study Group. Effect of community-based promotion of exclusive breastfeeding on diarrhoeal illness and growth: a cluster randomised controlled trial. Lancet. 2003; 361:1418-1423.

7. Coutsoudis, A., Pillay, K., Kuhn, L., et al. South African Vitamin A Study Group. Method of feeding and transmission of HIV-1 from mothers to children by 15 months of age: prospective cohort study from Durban, South Africa. AIDS. 2001; 15: 379-387.

8. World Health Organization. Breastfeeding and replacement feeding practices in the context of motherto- child transmission of HIV. An assessment tool for research. World Health Organization, Geneva, 2001. $\mathrm{WHO} / \mathrm{rhr} / 01.12 \mathrm{WHO} / \mathrm{CAH} / 01.21$

9. Magoni, M., Bassani, L., Okong P., et al. Mode of infant feeding and HIV infection in children in a program for prevention of mother-to- child transmission in Uganda. AIDS. 2005; 19: 433-437.

10. Iliff, P.J.,Piwoz, E.G., Tavengwa, N.V.et al.ZVITAMBO study group. Early exclusive breastfeeding reduces the risk of postnatal HIV-1 transmission and increases HIV-free survival. AIDS. 2005; 19: 699-708.

11. Ssenyonga, R., Muwonge, R. and Nankya, I. Towards a better understanding of exclusive breastfeeding in the era of HIV / AIDS: a study of prevalence and factors associated with exclusive breastfeeding from birth, in Rakai, Uganda. J. Trop. Pediatr. 2004; 50: 348-353.

12. Bland, R. M., Rollins, N.C., Coutsoudis, A. et al. Child Health Group. Breastfeeding practices in an area of high HIV prevalence in rural South Africa. Acta Paediatr. 2002; 91:704-711.

13. Gottlieb, D., Shetty, A.K., Mapfungautsi, R. M., et al. Infant feeding practices of HIVinfected and uninfected women in Zimbabwe. AIDS Patient Care STDS. 2004; 18: 45-53.

14. Matovu, A., Kirunda, B., Rugamba-Kabagambe, G., et al. Factors influencing adherence to exclusive breast feeding among HIV infected mothers in Kabarole district, Uganda. East Afr. Med. J. 2008; 85: 162-170.

15. Uganda Bureau of Statistics (UBOS) and ORC Macro. Uganda demographichealth survey 2000-2001. UBOS and ORC Macro. 2001.

16. Bland, R.M., Rollins, N.C., Solarsh, G. et al. Child Health Group. Maternal recall of exclusive breast feeding duration. Arch. Dis. Child. 2003; 88: 778-783. 